\title{
Endoscopic surgery in hemangiomas of paranasal sinuses and nasal cavity
}

\begin{abstract}
Objectives: To determine the possibility of performing complete resections of rhinosinusal hemangiomas greater than $2 \mathrm{~cm}$ treated by an endonasal approach assisted with endoscopes.

\section{Study design: Descriptive, retrospective}

Methods: We analyzed the clinical histories of all adult patients who underwent an endonasal approach with endoscopes, for having hemangiomas of the nasal cavity and paranasal sinuses in the Italian Hospital of Buenos Aires, between June 2006 and December 2016. We excluded patients with hemangiomas who were less than 18 years old, with small tumors (less than $2 \mathrm{~cm}$ ), vascular malformations, and those who underwent surgical procedures performed externally. The surgeries were performed under general anesthesia, with endoscopes of $0^{\circ}$ and $30^{\circ}$, conventional surgical instruments were used for rhinosinusal endoscopic surgery. The follow-up was performed by nasal endoscopy, computed tomography and/or magnetic resonance with contrast.
\end{abstract}

Results: Four patients were treated for nasal cavity (1/4) and paranasal sinuses (3/4) hemangiomas through an endonasal approach with endoscopes. There were no complications. The follow-up was greater than 5 years in two, 2 years in one and 1 year in another patient. No recurrences were detected.

Conclusión: Trans-nasal surgery assisted with endoscopes of paranasal sinus and nasal cavity hemangiomas greater than $2 \mathrm{~cm}$ in adults, allowed perform complete resections in $100 \%$ of treated patients. In extensive hemangiomas and when it was not possible to detect the site of vascular tumor implantation, it was important to previously devascularize the lesion by preoperative embolization and cauterization of the vascular pedicles before resecting the tumor.

Keywords: hemangioma, endoscopic surgery, paranasal sinus
Volume II Issue 3 - 2019

\author{
Carlos Santiago Ruggeri,' Guillermo \\ Sebastian Aragón,' Lautaro Acosta, ${ }^{2}$ Eduardo \\ Figueroa, ${ }^{2}$ \\ 'Rhinosinusology Division, Department of Otolaryngology, \\ Italian Hospital Buenos Aires, Argentina \\ ${ }^{2}$ Department of Otolaryngology, Italian Hospital Buenos Aires, \\ Argentina
}

\begin{abstract}
Correspondence: Carlos Santiago Ruggeri MD, Department of Otolaryngology, Italian Hospital Buenos Aires - Gascon 450, CPI I8I, CABA, Hospital Italiano de Buenos Aires, Argentina, Email carlos.ruggeri@hospitalitaliano.org.ar
\end{abstract}

Received: March 16, 2019 | Published: May 03, 2019

\section{Introductión}

Hemangiomas are benign neoplasms, common in the head and neck region, but very infrequent in the paranasal sinuses. They grow slowly and can be locally destructive due to the pressure exerted on the bone. These characteristics make them difficult to differentiate from malignant tumors originating in paranasal sinuses.

The main treatment of paranasal sinus hemangiomas in adults is surgery, being the transnasal approach with endoscopes alone or combined with an external approach the most used.

\section{Methods}

We analyzed the clinical histories of all adult patients who underwent an endonasal approach with endoscopes, for having hemangiomas of the nasal cavity and paranasal sinuses in the Italian Hospital of Buenos Aires, between June 2006 and December 2016. We excluded patients with hemangiomas who were less than 18 years old, with small tumors (less than $2 \mathrm{~cm}$ ), vascular malformations, and those who underwent surgical procedures performed externally. All were studied by nasal endoscopy, computed tomography and magnetic resonance with contrast. The surgeries were performed under general anesthesia, with endoscopes of $0^{\circ}$ and $30^{\circ}$, conventional surgical instruments were used for rhinosinusal endoscopic surgery. By endonasal approach a maxillary antrostomy, ethmoidectomy, and a middle turbinate resection were performed, and the tumor was resected. In another patient, the sphenopalatine artery was also cauterized, and a sphenoidotomy was performed, and in the remaining patient, the tumor of the nasal cavity was directly resected. The follow-up was performed by nasal endoscopy, computed tomography and/or magnetic resonance with contrast.

\section{Results}

Four patients were treated for nasal cavity (1/4) and paranasal sinuses (3/4) hemangiomas through an endonasal approach with endoscopes between June 2006 and December 2016, at the Hospital Italiano de Buenos Aires. Three were men and one woman, the youngest was 36 and the oldest 65 years, the average age was 46.6 years. The main reason for consultation was unilateral $(1 / 3)$, bilateral (1/3) nasal obstruction, and epistaxis (1/3). One patient also had epiphora, and proptosis was detected in two. In one of the patients, the hemangioma was a finding in a tomography requested to complete the evaluation for sleep apnea. Two tumors were located mainly in the maxillary sinus (one right and one left), displaced the eye and one had involved of the both nostrils and it spread to rhinopharynx. Another was located in the posterior ethmoid and another in the nasal cavity. In the 2 patients with giant tumors located in the maxillary sinus, endonasal biopsies were performed. One of the patients required a nasal tamponade due to significant bleeding.

The histopathological diagnosis was cavernous hemangioma, and in the other: complicated hemangioma with thrombosis and chronic inflammation. The other two patients underwent transnasal endoscopic resection and intraoperative histopathological study by freezing the surgical specimen. In the patient with the maxillary hemangioma, who presented an important epistaxis after the biopsy, a 
digital angiography with embolization was performed 48 hours before the surgery. In the 2 patients with hemangiomas of the maxillary sinus a medial maxillectomy, middle turbinectomy and anterior and posterior ethmoidectomy were performed (Figures 1\&2).

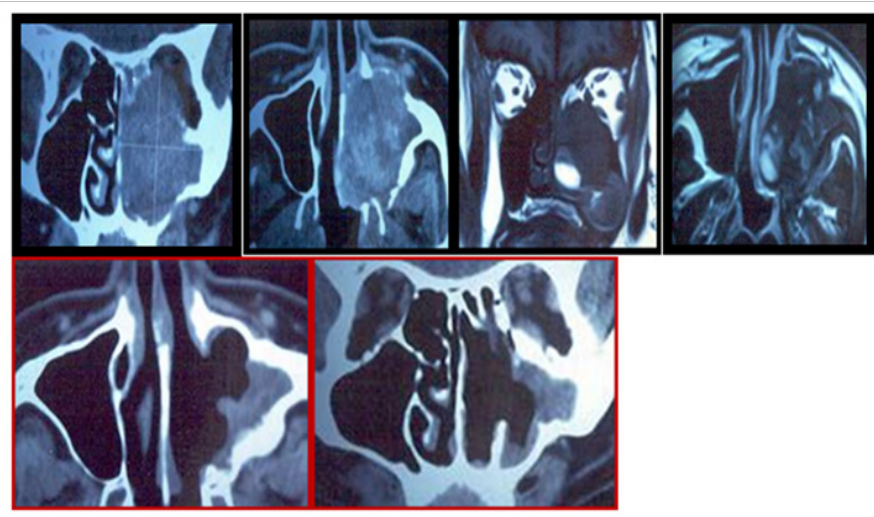

Figure I Maxillary sinus hemangioma treated by endonasal approach.
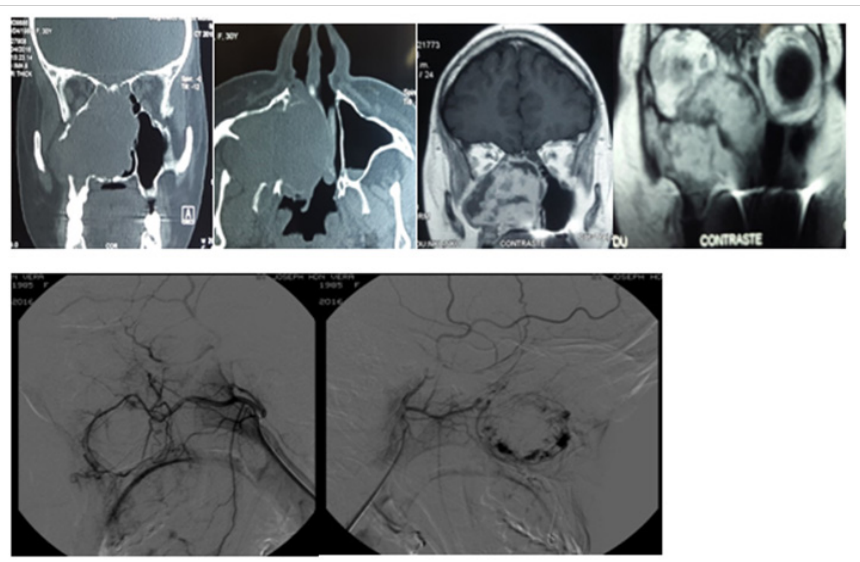

Figure 2 Maxillary sinus hemangioma treated by endonasal approach Embolization performed 48 hours prior to surgery.

It was not necessary to perform an external approach, nor did they receive blood transfusions during or after the surgery. The patient with the tumor located in the posterior ethmoid was operated by the endonasal approach and the vascular pedicle was controlled prior to the resection of the tumor by cauterizing the sphenopalatine artery (Figure 3).

The intraoperative histological study was reported as a vascular tumor without atypia and the deferred histopatological study as a cavernous hemangioma. The nasal cavity hemangioma was resected by endonasal approach. The intraoperative excisional biopsy was reported as a cavernous hemangioma (Figure 4). Two had a nasal tamponade that was removed at 48 hours and in 2 a hemostatic matrix was used (surgifló). The length of stay was 24 hours in three and 48 hours in another. There were no complications. The follow-up was greater than 5 years in two, 2 years in one and 1 year in another patient. No recurrences were detected.

\section{Discussión}

Hemangiomas are benign neoplasms composed of blood vessels, histologically there are three subtypes: capillary, cavernous and mixed hemangioma. The capillary hemangioma is a tumor formed by blood vessels that mostly have the caliber of normal capillaries, the lesions have a defined limit, but do not have a capsule, they consist of a tight accumulation of thin-walled capillaries separated by a sparse stroma of conjunctive tissue. One variant is the lobular capillary hemangioma, also called telangiectasic hemangioma or pyogenic granuloma. The latter term is inadequate because it is not constituted by a granulomatous infiltration nor is it produced by a microbial infection. There are several theories that try to explain their origin: traumatic lesions (digital or by nasal tamponades), hormonal (progesterone), viral influences, from underlying arteriovenous malformations or by production of angiogenic growth factors.
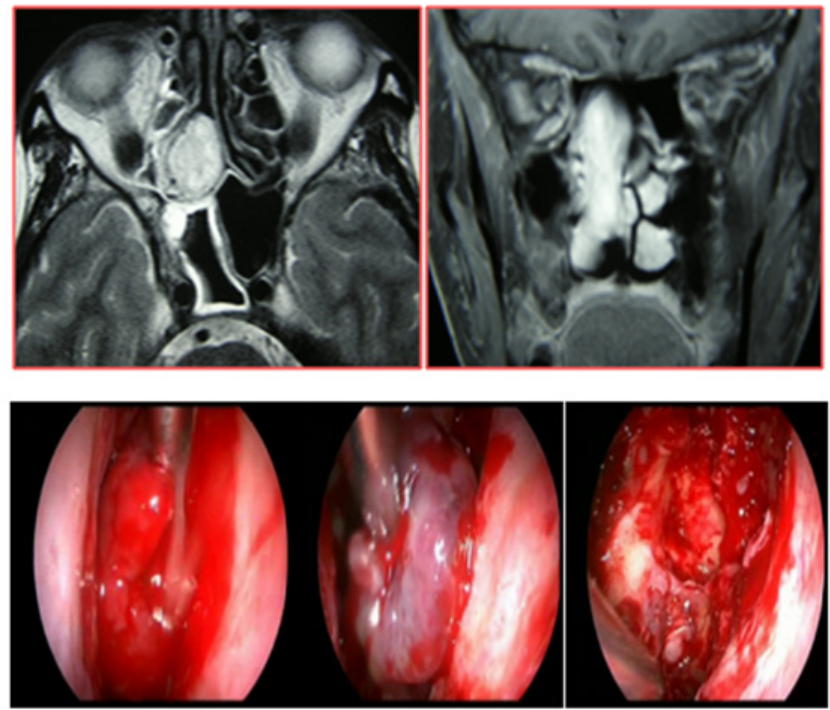

Figure 3 Posterior ethmoid hemangioma: endonasal assisted endoscopic resection.

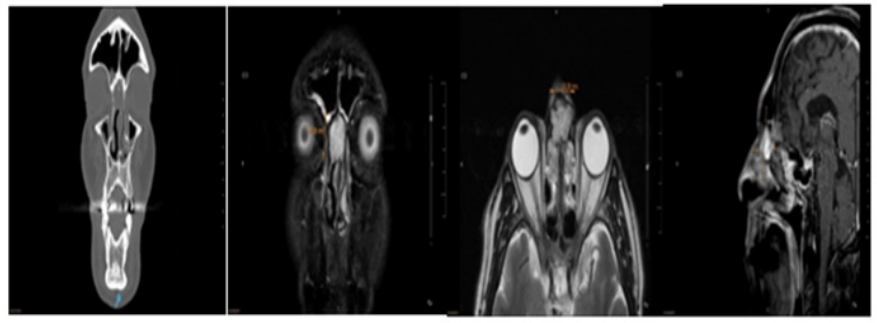

Figure 4 Nasal cavity hemangioma.

The cavernous hemangioma differs from the previous one due to the formation of larger vascular canals, in small lesions there may be one or two vascular channels, forming the entire lesion, it is not encapsulated (Figure 5). ${ }^{1}$ In a retrospective study, 1479 patients with hemangiomas of the whole body were included. Three hundred had hemangiomas in the head and neck region and only 37 patients had hemangiomas originating in the rhinosinusal mucosa. The sites of origin were: the nasal septum (40.5\%), inferior turbinate $(29.7 \%)$, maxillary sinus $(8.1 \%)$, uncinate process $(8.1 \%)$, middle turbinate $(2.7 \%)$, posterior ethmoid $(2.7 \%)$ and one originated on the nasal dorsum (2.7\%). Twenty-four were lobular capillary hemangiomas and 13 cavernous hemangiomas. The most common reason for consultation was nasal obstruction $(59.5 \%)$ and epistaxis $(51.4 \%){ }^{2}$ In our study, hemangiomas located in the maxillary sinus were more 
frequent and as in the Kim series the symptoms and signs of nasal obstruction and epistaxis were predominant.

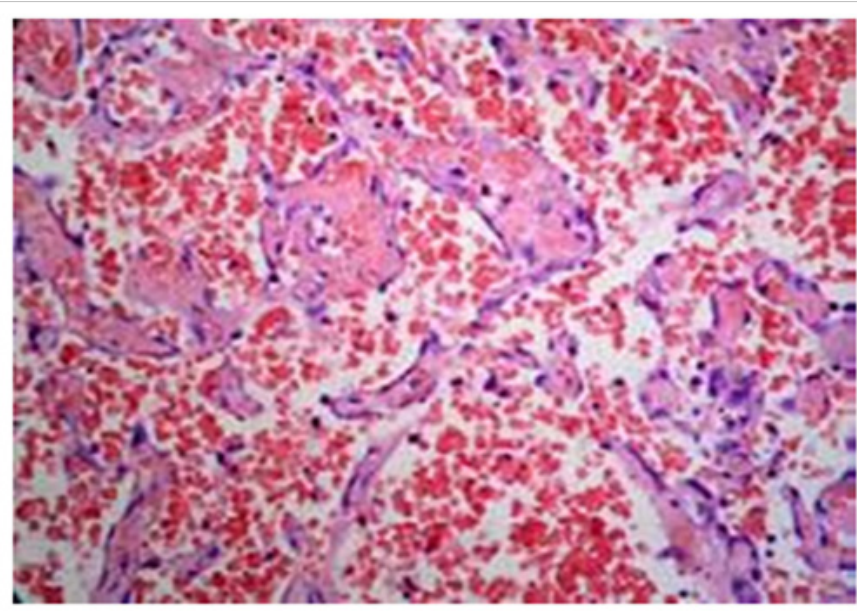

Figure 5 Cavernous hemangioma: Large vascular spaces with abundant erythrocytes surrounded by a connective.

In the two patients with giant hemangiomas located in the maxillary sinus, previous biopsies were performed due the destructive characteristics that raised a differential diagnosis with malignant lesions. One of them was referred to our hospital with a biopsy done in another center (required anteroposterior nasal tamponade). The sample review confirmed the diagnosis of cavernous hemangioma. In the other patient, it was decided to perform a biopsy prior to treatment to rule out a malignant tumor of the maxillary sinus, favored by the low degree of vascularization detected in the magnetic resonance with contrast.

\section{Individual treatment is based on tumor size, age and patient symptoms}

Corticosteroids may be useful to treat proliferative stage hemangiomas (children), where it is believed that they would block estradiol receptors and stop their growth. Propanolol is also used to treat childhood hemangiomas. In large paranasal sinus and nasal cavity hemangiomas we prefer to perform complete surgical resection using a transnasal approach under general anesthesia, with previous devascularization of the tumor by cauterization and /or embolization of the vascular pedicles that nourish the lesion.

It is convenient to resect the lesion in a subpericóndrico or subperiosteal plane. Intralesional therapy with bevacizumad (monoclonal antibody that inhibits the angiogenic pathway mediated by vascular endothelial growth factor) has been reported to be successful in treating recurrent hemangiomas. ${ }^{3}$ According to some studies ${ }^{4-5}$ the Nd: YAG laser can be used to facilitate the resection of hemangiomas in selected cases, its wavelength is preferentially absorbed by the hemoglobin, resulting in a deep penetration of approximately $1 \mathrm{~cm}$. The danger is that its penetration can cause thermal damage to deep tissues. It does not allow the coagulation of vessels larger than $3 \mathrm{~mm}$, or that have a high perfusion rate. It can be used percutaneously $(2 \mathrm{~W}$ at $25 \mathrm{~W}, 0.2-5 \mathrm{sec}$ ) or interstitially ( $4 \mathrm{~W}$ at $10 \mathrm{~W}, 10-120 \mathrm{sec}$ ), introducing the fiber of the laser into an intravenous catheter and penetrating the lesion. There are few studies in the literature that report the results of treatment in rhinosinusal hemangiomas in adults with an adequate number of patients, most of the works are presentations of clinical cases. In a study of 31 patients treated for rhinosinusal hemangiomas, all were operated endonasally with endoscopes. Small hemangiomas (N: 20, 8.3+-3.5mm) were treated under local anesthesia, and major hemangiomas $(\mathrm{N}: 11)$ of $20.5+-8.6 \mathrm{~mm}$ under general anesthesia. In 2, they used preoperative embolization. They reported a single recurrence $(3 \%)$ in a patient operated under local anesthesia during the eighth month of pregnancy. ${ }^{6}$

Pregnancy is considered to have an adverse effect on vascular injuries. The combination of estrogen and progesterone would increase venous distensibility, and stimulate endothelial proliferation, although no receptors for these hormones have been detected in 21 lobulated capillary hemangiomas. ${ }^{7-10}$ In another study, 2 recurrences were reported in 37 patients treated with endonasal surgery with endoscopes. ${ }^{3}$ In all the studies referred to above, they used local anesthesia to operate patients with smaller hemangiomas, which originated in the nasal cavity and in which they identified the implantation site. In our series, all hemangiomas were greater than 2 $\mathrm{cm}$ and only 1 was located in the nasal cavity, which is why they were treated under general anesthesia.

\section{Conclusión}

Trans-nasal surgery assisted with endoscopes of paranasal sinus and nasal cavity hemangiomas greater than $2 \mathrm{~cm}$ in adults, allowed perform complete resections in $100 \%$ of treated patients. In extensive hemangiomas and when it was not possible to detect the site of vascular tumor implantation, it was important to previously devascularize the lesion by preoperative embolization and/or cauterization of the vascular pedicles before resecting the tumor.

\section{Acknowledgment}

None.

\section{Conflicts of interest}

Authors declare that there is no conflict of interest.

\section{References}

1. Werner J, Dunne A, Folz B. Current Concepts in the Clasification , Diagnosis and Treatement of Haemangiomas and Vascular Malformation of the Head and Neck. Eur Arch Otorhinolaryngol. 2001;258(3):141-149.

2. Kim JS, Kwon SH. Sinonasal Hemangioma: Diagnosis, Treatment, and Follow-Up of 37 Patient at a Single Center. J Oral Maxillofac Surg. 2017;75(8):1-7.

3. Kinzinger Michael R, Strong E Bradley, Bernard Joan, et al. Intralesional Bevacizumab for the Treatment of Recurrent Sinonasal Hemangioma. Ann Otol Rhinol Laryngol. 2018;127(12):1-5.

4. Hackert I, Offergeld C. Lasers in Dermatology (Including Intersticial Therapy), in Lasers in Otorhinolaryngology. Karl-Bernd Huttenbrink. Thieme 2005:143-155.

5. Jochen A Werner MD, Burkard M Lippert MD, Stefan Gottschlich $\mathrm{MD}$, et al. Ultrasound-Guided Interstitial Nd: YAG Laser Treatment of Voluminous Hemangiomas and Vascular Malformations in 92 Patients. Laryngoscope. 1998;108(4):463-470.

6. Takaishi S, Asaka D, Nakayama T, et al. Features os Sinonasal Hemangioma: A retrospective Study of 31 Cases. Auris Nasus Larynx. 2017;44(6):1-5.

7. Nichols GE, Gaffey MJ, Mills SE, et al. Lobular capillary hemangioma. An immunohistochemical study including steroid hormone receptor status. Am J Clin Pathol.1992;97(6):770-775. 
8. Naser A, Samith A, Ríos C. Hemangioma cavernoso gigante de seno maxilar.Reporte de un caso y revisión de la literatura. Revista de Otorrinolaringología y Cirugía de Cabeza y Cuello. 2009;69(2):131136.

9. Roberto Puxeddu MD, Marco Berlucchi MD, Gian Peppino Ledda MD, et al. Lobular capillary hemangioma of the nasal cavity: A retrospective study on 40 patients. American Journal of Rhinology. 2006;20(4):480484.
10. Seth S, Kaur K, Bhardwar V. Cavernous Hemangioma of Maxillary Sinus. Iran J Radiol. 2012;3(1):42-44. 